\title{
Growth and characterization of gel grown pure and mixed iron-manganese levo-tartrate crystals
}

\author{
S J JOSHI, B B PAREKH, K D VOHRA ${ }^{\dagger}$ and M J JOSHI* \\ Department of Physics, Saurashtra University, Rajkot 360 005, India \\ ${ }^{\dagger}$ Publication Section, Gujarat University, Ahmedabad 380 009, India
}

MS received 23 February 2006; revised 18 April 2006

\begin{abstract}
Several applications of iron tartrate and manganese tartrate compounds are reported in the literature. In the present investigation, we have grown pure and mixed iron (II)-manganese levo-tartrate crystals by single diffusion gel growth technique. Crystals with spherulitic morphology were harvested. The colouration of the crystals changed from black to pinkish brown upon increasing the content of manganese in the crystals. The crystals were characterized by FTIR spectroscopy, powder XRD, TGA, VSM and dielectric study. Crystal structures of different mixed crystals were studied. From TGA it was observed that on heating the hydrated crystals became anhydrous and then converted into oxides. Paramagnetic nature of the crystals was revealed from VSM study. The variation of the dielectric constant with frequency was studied. The results are discussed.
\end{abstract}

Keywords. Mixed crystals; gel growth; FTIR; powder XRD; VSM; dielectric study; TGA.

\section{Introduction}

Several tartrate compounds deserve special attention due to their medical, pharmaceutical and industrial applications. For example, injections of $\mathrm{Na}-\mathrm{Cr}$ tartrate increase the susceptibility of transplanted sarcoma to the effect of X-rays (Veselaya and Gordyski 1957), calciphylatic responses of various ferrous tartrate compounds to prevent anemia in animals (Strebel et al 1962), ferrous tartrate as a catalyst in manufacture of champagne (Gvelesiani 1957), tanning action of ferrous tartrate to tan skin (Kubota 1959) and the use of manganese tartrate crystals in chemical temperature indicators (Gvozdov and Eronov 1958). The iron (II) tartrate crystals have been successfully grown by the gel growth technique and were characterized (Joseph and Joshi 1997; Joseph et al 1997a, b) using TGA, FTIR and Mössbauer spectroscopy. The aim of the present study is to grow pure and mixed iron (II)-manganese levo-tartrate crystals by the single diffusion gel growth technique and characterize them by several methods.

\section{Experimental}

The crystals were grown by the single diffusion gel growth technique. Glass test tubes of $25 \mathrm{~mm}$ diameter and $150 \mathrm{~mm}$ length were used as crystallization apparatus. Sodium

\footnotetext{
*Author for correspondence (mshilp24@ rediffmail.com)
}

metasilicate solution of specific gravity (range from 1.041.06) was acidified with levo-tartaric acid in such a way that the $\mathrm{pH}$ of the mixture could be set within $3 \cdot 6-4.0$ as per desired condition. The mixture was transferred into several glass test tubes. One molar aqueous solutions of ferrous sulphate and manganese chloride were poured in the following manner without disturbing the set gel: (a) $1 \mathrm{M}, 10 \mathrm{ml}$ $\mathrm{MnCl}_{2}$, (b) $1 \mathrm{M}, 8 \mathrm{ml} \mathrm{MnCl} 2+1 \mathrm{M}, 2 \mathrm{ml} \mathrm{FeSO}_{4}$, (c) $1 \mathrm{M}$, $6 \mathrm{ml} \mathrm{MnCl}_{2}+1 \mathrm{M}, 4 \mathrm{ml} \mathrm{FeSO}_{4}$, (d) $1 \mathrm{M}, 4 \mathrm{ml} \mathrm{MnCl}_{2}+$ $1 \mathrm{M}, 6 \mathrm{ml} \mathrm{FeSO}$, (e) $1 \mathrm{M}, 2 \mathrm{ml} \mathrm{MnCl} 2+1 \mathrm{M}, 8 \mathrm{ml} \mathrm{FeSO}$ and (f) $1 \mathrm{M}, 10 \mathrm{ml} \mathrm{FeSO}_{4}$. The following reaction was expected to occur

$$
\begin{aligned}
& (1-X) \cdot \mathrm{FeSO}_{4(\mathrm{aq})}+\mathrm{X} \cdot \mathrm{MnCl}_{2(\mathrm{aq})}+\mathrm{H}_{2} \mathrm{C}_{4} \mathrm{H}_{4} \mathrm{O}_{6} \rightarrow \\
& \quad \mathrm{Mn}_{x} \mathrm{Fe}_{(1-x)} \mathrm{C}_{4} \mathrm{H}_{4} \mathrm{O}_{6}+2 X \mathrm{HCl}+(1-X) \mathrm{H}_{2} \mathrm{SO}_{4}, \\
& \text { where } x=0,0 \cdot 2,0 \cdot 4,0 \cdot 6,0 \cdot 8 \text { and } 1 .
\end{aligned}
$$

Spherulitic crystals were grown within the gel. The growth was completed within a month. The colouration of pure Mn-tartrate was pinkish brown, which became darker upon adding iron and became dark blackish green in case of pure iron tartrate. Figures $1 \mathrm{a}$ and $\mathrm{b}$ are photographs exhibiting crystal growth for solution (d) and the as grown crystals removed from the test-tube, respectively. The composition of crystals was determined by EDAX, which is 24.74 at.\% $\mathrm{Mn}$ and 11.7 at.\% Fe; 9.86 at.\% $\mathrm{Mn}$ and 13.6 at. $\% \mathrm{Fe} ; 7.56$ at.\% $\mathrm{Mn}$ and 26.21 at.\% $\mathrm{Fe}$ as well as 5.95 at. $\% \mathrm{Mn}$ and 35.08 at.\% $\mathrm{Fe}$ for mixed crystal samples (b) to (e), respectively. 
(a)
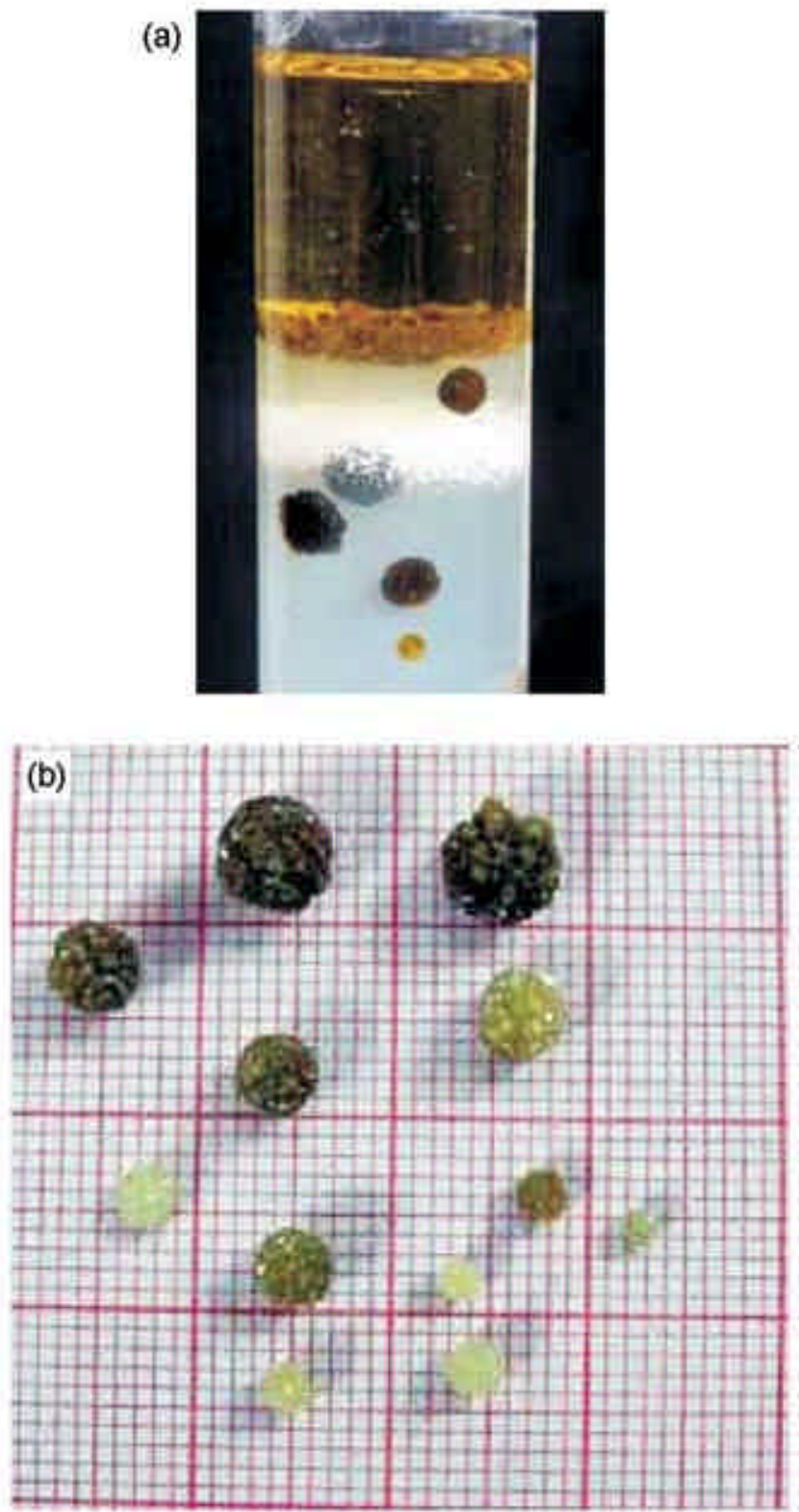

Figure 1. a. Growth of crystals for solution (d) in a gel and b. as grown spherulitic crystals.

\section{Characterization techniques}

The crystals were characterized by different analytical techniques. The FTIR spectra were recorded on BRUKER IFS 66V FT-IR spectrometer in the range from 400$4000 \mathrm{~cm}^{-1}$. Powder XRD was conducted by using PHILIPS X'PERT MPD system. The thermogravimetric analysis was performed on NETZSCH Geratebau $\mathrm{GmbH}$ from room temperature to $900^{\circ} \mathrm{C}$ at a heating rate of $15^{\circ} \mathrm{C} / \mathrm{min}$. The magnetic moment was measured for powdered samples using EG and PARC-155 VSM in the magnetic field range from 0-1.6 Tesla at room temperature. The dielectric measurements were carried out on powdered sample pellets at room temperature by using LCR meter model Agilent4284-A, in the frequency range from $500 \mathrm{~Hz}-1 \mathrm{MHz}$.

\section{Results and discussion}

Crystal growth by gel technique has attracted the attention of numerous researchers since it is useful to grow crystals at ambient temperatures. By remaining chemically inert, the gel framework acts like a three-dimensional crucible in which the crystal nuclei are delicately held in the position of their formation and supplied with the nutrients for growth (Henisch 1973). This technique has also attracted several researchers to grow different crystals for various applications (Henisch et al 1965; Patel and Arora 1977) and crystallization of urinary crystal (Joshi and Joshi 2003; Joseph et al 2005).

Figure 2 shows FTIR spectra of samples (a), (c) and (f). It can be observed from the spectra that the water of crystallization is associated with all crystals resulting into absorption within $3100-3600 \mathrm{~cm}^{-1}$. The carboxyl $(\mathrm{C}=\mathrm{O})$ group stretching vibrations are observed at slightly $<1600 \mathrm{~cm}^{-1}$. The $\mathrm{O}-\mathrm{H}$ deformation out of plane and $\mathrm{C}-\mathrm{H}$ stretching occur from $950-630 \mathrm{~cm}^{-1}$. This proves that the $\mathrm{O}-\mathrm{H}$ bond, the $\mathrm{C}=\mathrm{O}$ group and the $\mathrm{C}-\mathrm{H}$ bond are present. One can also see from figure 2 that as the content of iron increases the sharpness of absorption bands changes in the lower wave number ranges. Interference or perturbations are expected to shift characteristic bands due to (i) the electro negativity of neighbouring group of atoms, (ii) the spatial geometry of the molecule, or (iii) the mechanical mixing of vibrational modes (Socrates 1990). The atomic mass of $\mathrm{Mn}$ is 54.938 and Fe, 55.847, which causes slight alteration in the molecular geometry and mechanical vibrations and as a consequence it is reflected in the spectra. However, as per one study the low concentration of impurity in calcium tartrate tetrahydrate crystals does not affect much the FT-IR spectra (Sahaya and Mahadevan 2005).

The powder XRD patterns of samples (a), (c) and (f) are shown in figure 3 . The cell parameters are obtained by computer software Powder-X and given in table 1. All samples exhibit orthorhombic crystal structure. The difference between atomic radii of iron $(0.76 \AA)$ and manganese $(0 \cdot 80 \AA)$ is not large; therefore, some variations take place in the adoption of the new crystal structure. Moving from samples (a) to (c), the crystal structure is somewhat nearer to that of $\mathrm{MnC}_{4} \mathrm{H}_{4} \mathrm{O}_{6}$, but as the amount of iron increases with some variations the crystals try to adopt to the crystal structure of $\mathrm{FeC}_{4} \mathrm{H}_{4} \mathrm{O}_{6}$.

Thermal studies of iron (II) tartrate were reported (Joseph et al 1997a) using thermogravimetry. Different kinetic and thermodynamic parameters from the thermograms were evaluated (Joseph and Joshi 1997). Recently, thermogravimetry of pure and impurity added calcium tartrate tetrahydrate crystals were reported (Sahaya and Maha- 


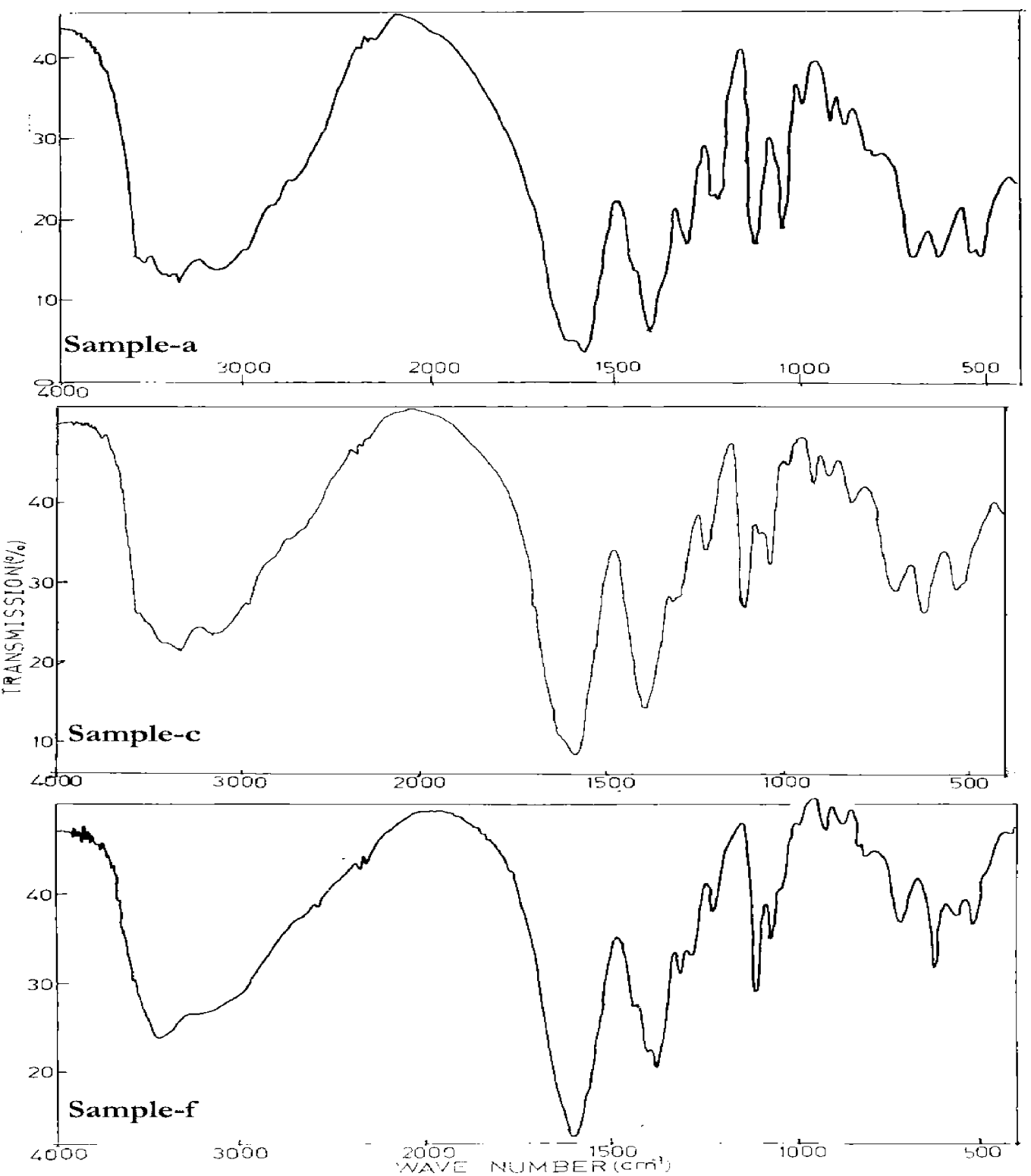

Figure 2. FTIR spectra of samples (a), (c) and (f).

Table 1. The crystal structure, water of hydration and bulk magnetic susceptibility data.

\begin{tabular}{lccccc}
\hline & \multicolumn{3}{c}{ Crystal structure parameter } & & \\
\cline { 2 - 4 } Sample name & $a(\AA)$ & $b(\AA)$ & $c(\AA)$ & Water of hydration & $\begin{array}{r}\text { Magnetic susceptibility }(\chi) \\
\left(\times 10^{-2} \mathrm{~A} \mathrm{~m}^{2} / \mathrm{kg} \mathrm{T}\right)\end{array}$ \\
\hline Sample (a) & 9.7559 & $11 \cdot 2290$ & $6 \cdot 2278$ & $1.5 \mathrm{H}_{2} \mathrm{O}$ & $70 \cdot 70$ \\
Sample (b) & 9.7897 & 11.1000 & $6 \cdot 1997$ & $2 \mathrm{H}_{2} \mathrm{O}$ & $52 \cdot 10$ \\
Sample (c) & $8 \cdot 8570$ & 11.0357 & $7 \cdot 5000$ & $1.5 \mathrm{H}_{2} \mathrm{O}$ & $46 \cdot 18$ \\
Sample (d) & 8.9200 & 10.6990 & $8 \cdot 1989$ & $2 \mathrm{H}_{2} \mathrm{O}$ & $45 \cdot 88$ \\
Sample (e) & 8.8810 & 11.1290 & $8 \cdot 1800$ & $2.5 \mathrm{H}_{2} \mathrm{O}$ & 50.44 \\
Sample (f) & 8.7588 & 10.9889 & $8 \cdot 1900$ & $2.5 \mathrm{H}_{2} \mathrm{O}$ & $45 \cdot 80$ \\
\hline
\end{tabular}

devan 2005). Thermograms of samples (a), (c) and (f) are shown in figure 4 . Figure 4 suggests that upon heating the samples, first loss of water of hydration and water of crystallization occurs and, thereafter, decomposition into oxides takes place. In the case of sample (a) the decomposition to manganese oxide is through smooth single 

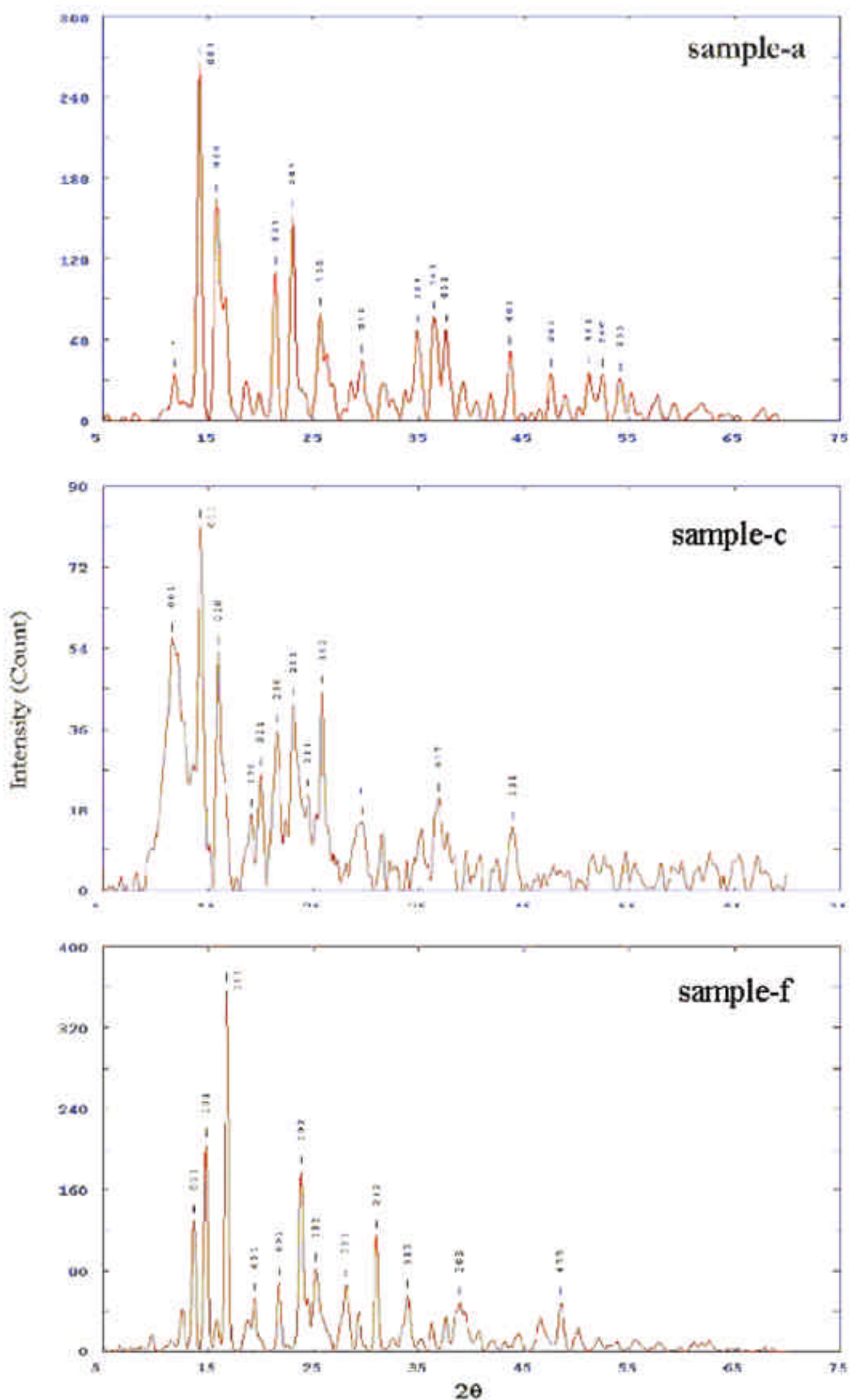

Figure 3. Powder XRD of samples (a), (c) and (f). 

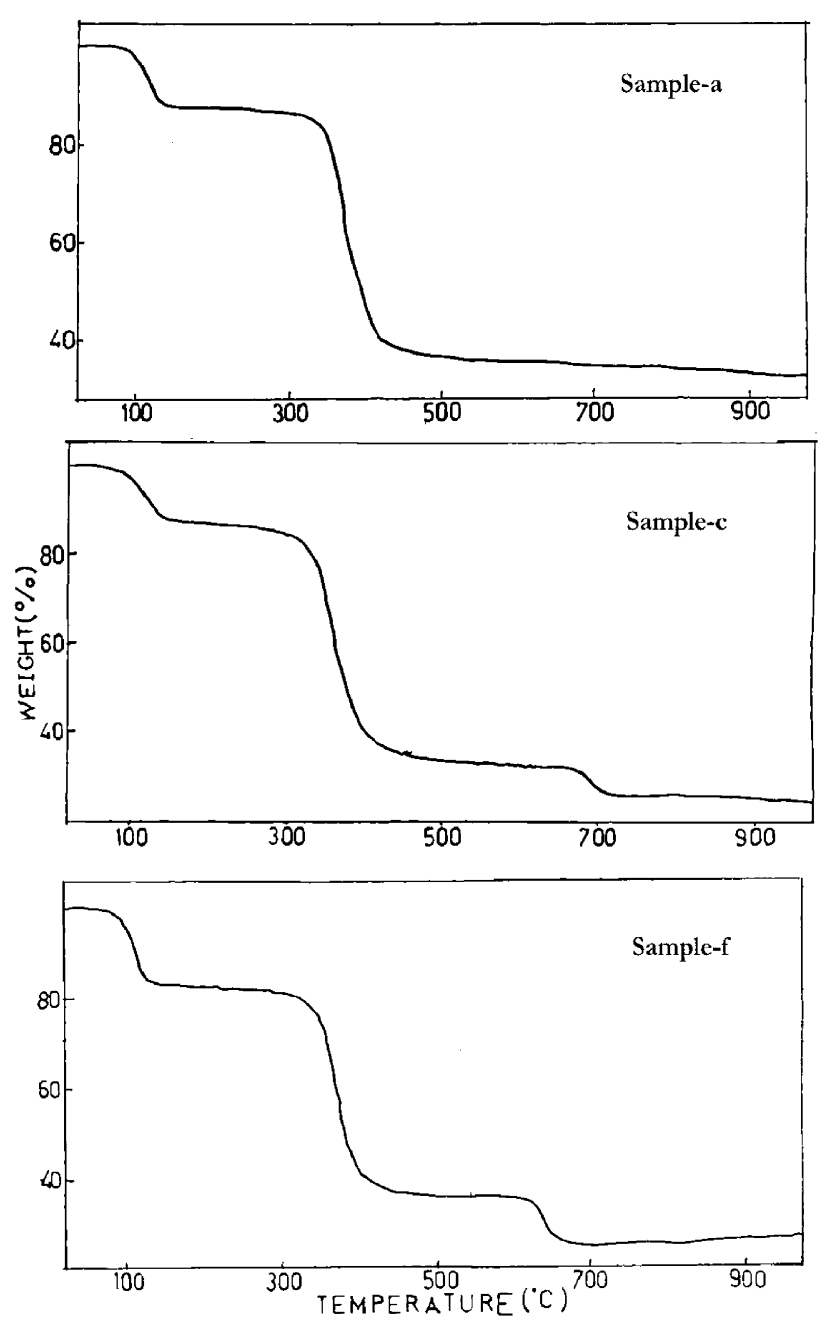

Figure 4. Thermograms of samples (a), (c) and (f).

step, while for samples (c) and (f) the transition to mixed $\mathrm{Mn}-\mathrm{Fe}$ oxide and Fe-oxide, respectively, is through one intermediate unstable state of oxide. The samples are thermally unstable and lose water of hydration upon heating. Also, the temperature of formation of final compound decreases on increasing iron in the crystals. The percentage values of weight losses for various samples were calculated for different stages of thermograms and were found to be matching closely with those of the observed ones. Water of hydration for different crystals have been calculated and compiled in table 1 .

It has been considered that the magnetic susceptibility data of ferrous tartrate suggested an octahedral environment around Fe-atom (Patel et al 1997). From the estimated magnetic susceptibility value of iron tartrate (5.4 BM), it has been conjectured that an octahedral environment around $\mathrm{Fe}(\mathrm{II})$ prevails (Mabbe and Machhin 1973) and later on a molecular formula, $\mathrm{FeC}_{4} \mathrm{H}_{4} \mathrm{O}_{6} \cdot 5 \mathrm{H}_{2} \mathrm{O}$, has been suggested, so that six coordination of $\mathrm{Fe}(\mathrm{II})$ is satisfied (Patel et al 1997). Earlier, Mn(II), Cu(II) and Ni(II) complexes with lactic acid, malonic acid and tartaric acid were prepared with standard technique and their magnetic susceptibilities were studied by using magnetic balance (Ranade and Subba Rao 1966). Moreover, the magnetic properties of manganese tartrate dihydrate crystals were reported by using VSM (Bhatt 1997). However, a weak ferromagnetism in manganese tartrate dihydrate single crystals was reported (Paduan-Filaho and Becerra 2000). In the magnetic susceptibility study, from room temperature to $0.4^{\circ} \mathrm{K}$ in the presence of magnetic field of $70 \mathrm{kOe}$, the antiferromagnetic order below $T_{\mathrm{N}}=138 \mathrm{~K}$, with spin or iented close to the $c$-axis was reported. The sharp peaks in susceptibility and the presence of a remnant magnetization in the plane perpendicular to the $c$-axis suggested a weak ferromagnetic structure of spins with canting angle $0 \cdot 6^{\circ}$. The magnetic properties were studied, in the present investigation, through the data of magnetic moment at different applied magnetic fields, which were obtained on the VSM. The bulk magnetic susceptibility was calculated. All samples were found to be paramagnetic. Table 1 gives the data of magnetic susceptibility. No systematic variation was observed in the magnetic susceptibility; however, Mn-tartrate exhibited the maximum value of susceptibility.

The dielectric constant was determined from the value of capacitance, area and thickness of the pellet of powdered samples in usual manner. The variation of dielectric constant with frequency is shown in the plots of figure 5 for different samples. The dielectric constant decreases with increase in frequency. The electronic exchange of the number of ions in the crystals gives local displacement of electrons in the direction of applied field, which gives the polarization. As the frequency increases, a point is reached where the space charge cannot sustain and comply with the external field. Therefore, polarization decreases and exhibits reduction in the values of dielectric constant with increase in frequency. However, this occurs suddenly at $10 \mathrm{kHz}$ frequency, which indicates a sudden change in polarization. Also, it has been found that the variation of dielectric constant with frequency is not high and unlike in the other cases (Hiremath and Venkataraman 2003; Arora et al 2004; Dabhi et al 2005), it is not smooth but sudden transition is observed. Recently, the dielectric study has been reported on $\gamma-\mathrm{Fe}_{2} \mathrm{O}_{3}$ (Hiremath and Venkataraman 2003), Zn-tartrate crystals (Dabhi et al 2005) and strontium tartrate crystals (Arora et al 2004), which exhibit smooth decrease in the value of dielectric constant with increase in frequency. Further work is under progress.

\section{Conclusions}

Spherulitic crystals of pure and mixed Mn-Fe tartrate exhibit variation in colour on changing the content of iron and manganese. FTIR spectroscopic study suggests the presence of water of hydration, $\mathrm{C}=\mathrm{O}$ and $\mathrm{C}-\mathrm{H}$ bonds. Powder XRD suggests orthorhombic crystal structure. On increasing the content of iron in mixed crystals, a sudden flip towards 


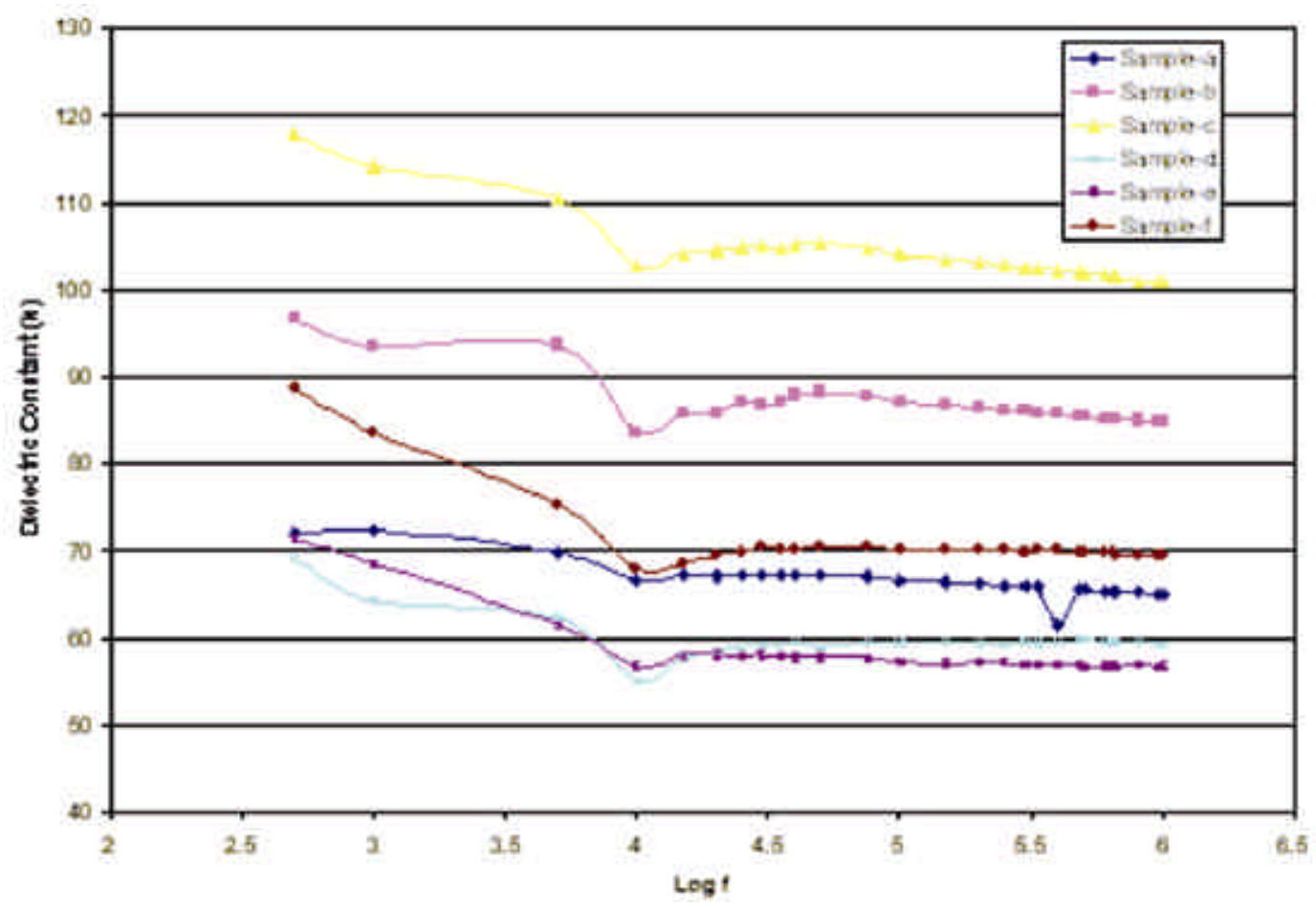

Figure 5. The plots of dielectric constant vs frequency for samples (a) to (f).

iron tartrate structure is observed. The crystals are thermally unstable; on heating they become anhydrous and then decompose into metallic oxides. The amount of water of hydration is determined. On increasing the amount of iron, the temperature of metal oxide stage formation of the compounds decreases and an intermediate stage of metal oxide becomes visible in the thermograms. All crystals exhibit paramagnetic nature. The dielectric constant decreases slowly on increasing the frequency, suggesting higher space charge polarizability in the low frequency region. However, a sudden change is observed at $10 \mathrm{kHz}$ frequency in this behaviour, which suggests a sudden change in polarization.

\section{Acknowledgements}

Authors are thankful to UGC for SAP grants and one of the authors (SJJ) thanks UGC for the minor research project. Authors are thankful to Prof. K N Iyer for his encouragement and Prof. H H Joshi and Dr K B Modi for their kind cooperation.

\section{References}

Arora S K, Patel V, Amin B and Kothari A 2004 Bull. Mater. Sci. 27141

Bhatt S N 1997 Gel growth of manganese tartrate crystals M Phil Dissertation, Saurashtra University, Rajkot

Dabhi R M, Parekh B B and Joshi M J 2005 Indian J. Phys. 79 503
Gvelesiani V P 1957 Vinodelie I Vinogro darstavo SSSR 1713 Gvozdov S P and Eronov A A 1958 Zavedenhil Khim. Teckhnol. 5154

Henisch H K 1973 Crystal growth in gels (University Park, Ohio: The Pennsylvania State Univ. Press)

Henisch H K, Dennis J and Hanoka J I 1965 J. Phys. Chem. Solids 26493

Hiremath V A and Venkataraman A 2003 Bull. Mater. Sci. 26 391

Joseph S and Joshi M J 1997 Indian J. Phys. A71 183

Joseph S, Joshi H S and Joshi M J 1997a Cryst. Res. Technol. 32339

Joseph S, Joshi H H and Joshi M J 1997b Indian J. Phys. A71 643

Joseph K C, Parekh B B and Joshi M J 2005 Curr. Sci. 881232

Joshi V S and Joshi M J 2003 Cryst. Res. Technol. 38817

Kubota M 1959 J. Chem. Soc. Jap. Ind. Chem. Sect. 57594

Mabbe F E and Machhin D J 1993 Magnetism and transition metal complexes (London: Chapman and Hall) p. 159

Paduan-Filaho A and Becerra C C 2000 J. Phys.: Cond. Matter 122071

Patel A R and Arora S K 1977 J. Cryst. Growth 37343

Patel M C, Ray A and Venkataraman A 1997 J. Phys. Chem. Solids $\mathbf{5 8} 749$

Ranade A C and Subba Rao V V 1966 Indian J. Chem. 442

Sahaya Shajan and Mahadevan C 2005 Cryst. Res. Technol. 40 598

Socrates G 1990 Infrared characteristic group frequencies (Chichester: John-Wiley)

Strebel R, Vasku J and Selye H 1962 J. Pharm. Pharmacol. 14 658

Veselaya I V and Gordyski V I 1957 Voprosy Onkol. 3300 